\title{
A Kalman decomposition to detect temporal linear system stucture
}

\author{
L.G. Van Willigenburg, W.L. De Koning ${ }^{1}$
}

\begin{abstract}
Feedback controllers for non-linear systems are often based on a linearized dynamic model. Such a linearized model may be temporarily uncontrollable and/or unreconstructable. This paper introduces the so-called differential Kalman decomposition of time-varying linear systems. It is based on differential controllability and differential reconstructability in conjunction with a linear time-varying continuous-time system description that allows the system structure and dimensions to change at certain timeinstants. We show how these together enable the detection of what will be called temporal system structure. This structure among other things reveals the temporal loss of controllability and/or reconstructability. Moreover this paper shows how time-varying state-dimensions enable a satisfactory realization theory for time-varying linear systems and how our Kalman decomposition is linked to the conventional ones.
\end{abstract}

\section{INTRODUCTION}

A general approach to control non-linear systems is to compute an optimal control and state trajectory offline using a non-linear systems model. To accommodate for disturbances the linearised dynamic model about these trajectories is used to design e.g. a linear quadratic perturbation feedback controller that operates on-line [1]. This approach depends critically on the controllability and reconstructability of the linearised dynamic model that is generally time-varying. If the systems model or the optimal control is not sufficiently smooth, e.g. if the control is bangbang, partly singular or digital, the time-varying linearised dynamic model may be temporarily uncontrollable and/or unreconstructable [2]. This implies that over the associated time-intervals the feedback controller is partly ineffective and the system may become unstable. Therefore the detection of temporal uncontrollability and unreconstructability is highly important to control engineers.

Most of linear systems theory and control system design is concerned only with the properties controllability,

${ }^{1}$ L. G. Van Willigenburg is with the Systems and Control Group of Wageningen University, P.O. Box 17, 6700 AA, Wageningen, The Netherlands (corresponding author, phone: +31 317 482941; fax: +31 317 484957; e-mail: Gerard.vanwilligenburg@ @ur.nl).

W.L. De Koning has retired and lives at Kroeskarper 6, 2318 NG, Leiden, The Netherlands (e-mail: wilros@ planet.nl). reachability, reconstructability and observability. These properties can be detected from Kalman decompositions of the linear system. As demonstrated in this paper these decompositions do not detect the temporal loss of such properties that is associated with temporal changes in the system structure. In this paper continuous-time systems with variable structure and dimensions are introduced along with the so-called differential Kalman decomposition. Together these enable the detection of what is called temporal system structure. Among other things this structure reveals immediately the temporal loss of familiar system properties.

\section{ILLUSTRATIVE EXAMPLE}

\section{Example 1}

Consider the following time-varying linear system,

$$
\begin{aligned}
& \dot{x}(t)=A(t) x(t)+B(t) u(t) \\
& y(t)=C(t) x(t), t \in[0,1]
\end{aligned}
$$

where

$$
\begin{aligned}
& A(t)=\left[\begin{array}{ll}
1 & 2 \\
1 & 1
\end{array}\right], t \in[0,0.25], A(t)=\left[\begin{array}{ll}
1 & 2 \\
0 & 1
\end{array}\right], t \in(0.25,0.5] \\
& A(t)=\left[\begin{array}{ll}
1 & 0 \\
2 & 1
\end{array}\right], t \in(0.5,0.75], A(t)=\left[\begin{array}{ll}
1 & 1 \\
2 & 1
\end{array}\right], t \in(0.75,1] \\
& B(t)=\left[\begin{array}{ll}
1 & 0
\end{array}\right]^{T}, C(t)=\left[\begin{array}{ll}
1 & 0
\end{array}\right], t \in[0,1]
\end{aligned}
$$

If time in (1) would be restricted to $(0.25,0.5)$ the system would be unreachable as well as uncontrollable. Similarly if time in (1) would be restricted to $(0.5,0.75)$ the system would be unobservable and unreconstructable. If according to (1) $t \in[0,1]$ then we might call the system temporarily uncontrollable/unreachable over $(0.25,0.5)$ because the second state variable is not influenced by the input. Similarly we might call the system temporarily unreconstructable/unobservable over $(0.5,0.75)$ because the second state variable does not influence the output. Since moreover the second state variable is unstable it cannot be stabilized by a controller over these time intervals. If we apply a similarity transformation at every time $t \in[0,1]$ to the system description (1)-(2) then the facts stated above are unchanged but no longer obvious 
from the system description. In this paper we will demonstrate that these facts will not become obvious either after application of any of the four conventional Kalman decompositions [3]. However they do become obvious after application of our differential Kalman decomposition that retrieves a system description similar to (1)-(2).

\section{CONTINUOUS-TIME SYSTEMS WITH VARIABLE STRUCTURE AND DIMENSIONS}

Quoting Kalman from [4]: "The only possibility of getting a reasonably well-rounded realization theory is to generalize the notion of a dynamical system in such a way that the dimension of the state-space is allowed to vary with time". Remarkably, except for [5], continuous-time systems with variable dimensions seem to have been ignored. A reason for this might be that a general description of timevarying dimensions and system structure requires the following system description that is uncommon,

$$
\begin{aligned}
& \dot{x}(t)=A(t) x(t)+B(t) u(t), y(t)=C(t) x(t), \\
& x(t) \in R^{n_{i}}, u(t) \in R^{m_{i}}, y(t) \in R^{l_{i}}, \\
& t \in\left(t_{i}, t_{i+1}\right), i=0,1, . ., N-1, \\
& x\left(t_{i}^{+}\right)=A_{i} x\left(t_{i}^{-}\right), A_{i} \in R^{n_{i+1} \times n_{i}}, i=1,2, . ., N-1 .
\end{aligned}
$$

In equation (3) $x$ denotes the state, $u$ the input that is assumed to be bounded and $y$ the output. Over every separate open interval $\left(t_{i}, t_{i+1}\right)$ equation (3) equals a conventional linear system description since $A(t), B(t)$ and $C(t)$ have constant dimensions. Moreover the controllability and reconstructability matrices are assumed to have a constant rank. As we shall see later in this paper this implies that the structure of the system is constant. Changes of the system structure and dimensions may only occur at the time instants $t_{i}, i=1,2, . ., N-1$. At these time instants the additional system matrices $A_{i}, i=1,2, . ., N-1$ describe the state transitions from $t_{i}^{-}$to $t_{i}^{+}$where the superscripts,-+ denote respectively the right and left time limit. In equation (3) $x\left(t_{0}^{+}\right)$should be identified as the initial state. Similarly $x\left(t_{N}^{-}\right)$should be identified as the terminal state. According to [6] we may call the system (3) a piecewise constant rank system (PCR system). The class of piecewise constant rank systems is very broad and contains among others piecewise time-invariant and piecewise analytic systems [6]. A PCR system differs from the switched linear systems in [7], [8] because the timeinstants $t_{i}, i=1,2, . ., N-1$ are a-priori fixed and the linear system over each time-interval $\left(t_{i}, t_{i+1}\right)$ is time-varying. The time domain of a PCR system is denoted by $\mathrm{T}$ given by,

$$
\mathrm{T}=\bigcup\left(t_{i}, t_{i+1}\right), i=0,1, . ., N-1
$$

Three types of PCR systems will be considered with different time domains. We consider PCR systems with $t_{0}=-\infty, t_{N}=+\infty$, with $t_{0}=0, t_{N}=+\infty$ and with, $t_{0}=0$, $t_{N}<+\infty$. These are denoted by,$-+ 0+$ and $0 \mathrm{~N}$ PCR systems respectively. In all the definitions in this paper concerning ,$+ 0+$ and $0 \mathrm{~N}$ PCR systems time should always be considered restricted to the associated time domain of the PCR system.

In practice we often start from a continuous-time system description with constant dimensions defined over $\left[t_{0}, t_{N}\right]$ such as example 1. Example 1 is almost everywhere equivalent to (3) if we select $t_{0}=0, t_{1}=0.25, t_{2}=0.5$, $t_{3}=0.75, t_{4}=t_{N}=1, A_{1}=A_{2}=A_{3}=I$, where $I$ denotes the identity matrix. In general we should select $t_{i}$, $i=1,2, \ldots, N-1$ as the times where the system description changes from one constant rank system to another and $A_{i}=I, \quad i=1,2, . ., N-1$. To do this we need to be able to detect these changes.

Let $s \in\left(t_{j}, t_{j+1}\right), t \in\left(t_{k}, t_{k+1}\right), t>s, 0 \leq j \leq k \leq N$. Then the state transition matrix $\Phi(t, s)$ of a PCR system satisfies,

$$
\begin{aligned}
& \Phi(t, s)=\Phi^{\prime}(t, s), k=j \\
& \Phi(t, s)=\Phi^{\prime}\left(t, t_{j+1}^{+}\right) A_{j+1} \Phi^{\prime}\left(t_{j+1}^{-}, s\right), k=j+1, \\
& \Phi(t, s)=\Phi^{\prime}\left(t, t_{k}^{+}\right) A_{k} \ldots \Phi^{\prime}\left(t_{j+2}^{-}, t_{j+1}^{+}\right) A_{j+1} \Phi^{\prime}\left(t_{j+1}^{-}, s\right), \\
& k>j+1 .
\end{aligned}
$$

In equation (5) the state transition matrices $\Phi^{\prime}$ on the right are conventional state transition matrices for continuous-time linear systems with constant dimensions. The Kalman decomposition computes and uses similarity transformations to decompose the system at any time. For a PCR system these are described by,

$$
\begin{aligned}
& x^{\prime}(t)=T(t) x(t), T(t) \in R^{n_{i} \times n_{i}}, \operatorname{rank}(T(t))=n_{i}, \\
& A^{\prime}(t)=T(t) A(t) T^{-1}(t)+\dot{T}(t) T^{-1}(t), \\
& B^{\prime}(t)=T(t) B(t), C^{\prime}(t)=C(t) T^{-1}(t), t \in\left(t_{i}, t_{i+1}\right), \\
& A_{i}^{\prime}=T\left(t_{i}^{+}\right) A_{i} T^{-1}\left(t_{i}^{-}\right), i=1,2, . ., N-1 .
\end{aligned}
$$

In equation (6) the prime denotes quantities associated with the system obtained after the similarity transformation $T(t), t \in\left(t_{i}, t_{i+1}\right), i=0,1, . ., N-1$.

\section{Definition 1}

Two PCR systems are called equivalent if one system can be obtained from the other through a similarity transformation $T(t), t \in \mathrm{T}$. Over each interval $\left(t_{i}, t_{i+1}\right)$, $i=0,1, . ., N-1, T(t)$ may be non-smooth at a finite number of isolated times. Every time non-smoothness occurs the 
interval $\left(t_{i}, t_{i+1}\right)$ is split up

\section{Definition 2}

The input-output map $G_{t_{0}, t_{N}}: U_{t_{0}, t_{N}} \mapsto Y_{t_{0}, t_{N}}$ of a PCR system maps any input sequence $U_{t_{0}, t_{N}}=\left\{u(t), t_{0}<t<t_{N}\right\}$ to the associated output sequence $Y_{t_{0}, t_{N}}=\left\{y(t), t_{0}<t<t_{N}\right\}$ as determined by equation (3) and in addition the initial state $x\left(t_{0}^{+}\right)$if the system is of the type $0+$ or $0 \mathrm{~N}$. Two PCR systems are called input-output equivalent if they have identical input-output maps almost everywhere on $\left(t_{0}, t_{N}\right)$.

\section{Definition 3}

A PCR system with dimensions $n(t)$ is called minimal if no PCR system with dimensions $n^{\prime}(t)$ has the same inputoutput map almost everywhere on $\left(t_{0}, t_{N}\right)$ and the property that $n^{\prime}(t) \leq n(t)$ almost everywhere on $\left(t_{0}, t_{N}\right)$ and $n^{\prime}(t)<n(t)$ over some open interval inside $\left(t_{0}, t_{N}\right)$. If a PCR system is minimal it is called a minimal realization of its associated input-output map and of any other PCR system with the same input-output map almost everywhere on $\left(t_{0}, t_{N}\right)$.

\section{Definition 4}

A PCR system is called reachable at time $t$ if there exists an $s<t$ such that any state $x(s)$ can be transferred to any state $x(t)$ through an appropriate choice of the input $U_{s, t}$. A PCR system is called differentially reachable ( $d$ reachable) at time $t$ if $s$ can be selected arbitrarily close to $t$. A PCR system (3) is called controllable from time $s$ if there exists a $t>s$ such that any state $x(s)$ can be transferred to any state $x(t)$ through an appropriate choice of the input. A PCR system (3) is called differentially controllable (d-controllable) from time $s$ if $t$ can be selected arbitrarily close to $s$.

\section{Definition 5}

A PCR system (3) is called observable at time $s$ if there exists a $t>s$ such that the state $x(s)$ can be determined from the output $Y_{s, t}$. A PCR system is called $d$-observable at time $s$ if $t$ can be selected arbitrarily close to $s$. A PCR system is called reconstructable from time $t$ if there exists an $s<t$ such that $x(s)$ can be determined from the output $Y_{s, t}$. A PCR system is called $d$-reconstructable from time $t$ if $s$ can be selected arbitrarily close to $t$.

\section{Definition 6}

The reachability/controllability grammian $W_{s, t}, t>s$, of the PCR system is given by,

$$
\begin{gathered}
\frac{d W_{s, t}}{d t}=A(t) W_{s, t}+W_{s, t} A^{T}(t)+B(t) B^{T}(t), t, s \in \mathrm{T}, t>s, \\
W_{s, t_{i}^{+}}=A_{i} W_{s, t_{i}^{t}} A_{i}^{T}, i=1,2, . ., N-1, s \in \mathrm{T}, s<t_{i}^{-}, W_{s, s}=0 .
\end{gathered}
$$

The transition rule (8) of $W_{s, t}$ from $t=t_{i}^{-}$to $t=t_{i}^{+}$equals the discrete-time rule without an input. This follows from the last line of equation (3). The observability/ reconstructability grammian $M_{s, t}, t>s$, of the PCR system is given by,

$$
\begin{gathered}
-\frac{d M_{s, t}}{d s}=A^{T}(s) M_{s, t}+M_{s, t} A(s)+C^{T}(s) C(s), t, s \in \mathrm{T}, t>s, \\
M_{t_{i}^{-}, t}=A_{i}^{T} M_{t_{i}^{+}, t} A_{i}, i=1,2, . ., N-1, t \in \mathrm{T}, t>t_{i}^{+}, M_{t, t}=0 .
\end{gathered}
$$

\section{Lemma 1}

For a PCR system and $s, t \in \mathrm{T}, t>s$ the following equivalence holds: any state $x(s)$ can be transferred to any state $x(t)$ through an appropriate choice of the input $U_{s, t}$ $\Leftrightarrow W_{s, t}>0$. For a PCR system and $s, t \in \mathrm{T}, t>s$ the following equivalence holds: the state $x(s)$ can be recovered from the output $Y_{s, t} \Leftrightarrow M_{s, t}>0$.

\section{Proof}

For continuous-time linear systems with constant dimensions the above lemma is well known. The discrete time transition rules at the times $t_{i}, i=1,2, . ., N-1$, do not change this because lemma 1 also holds in discrete-time, even if the dimensions of the discrete-time system are variable [7]

According to definition 4 and lemma $1, W_{s, t}>0$ implies that the system is reachable at time $t$ as well as controllable from time $s$. Therefore $W_{s, t}$ is called the reachability/controllability grammian in definition 6 and dually $M_{s, t}$ is called the observability/reconstructability grammian.

\section{Lemma 2}

Over each time-interval $\left(t_{i}, t_{i+1}\right), i=0,1, . ., N-1$, a PCR system is either d-reachable from/d-controllable at any time $t \in\left(t_{i}, t_{i+1}\right)$ or at no time $t \in\left(t_{i}, t_{i+1}\right)$. A dual result applies to d-observability/d-reconstructability.

\section{Proof}

Over each time-interval $\left(t_{i}, t_{i+1}\right), \quad i=0,1, . ., N-1$, the situation is comparable to the one for conventional constant 
rank systems. Then the result follows from [6]

\section{THE DIFFERENTIAL KALMAN DECOMPOSITION}

Kalman decomposition are introduced and applied in this section to decompose PCR systems and detect global and local system structure.

Given the modifications and definitions presented in the previous section, procedures to compute the Kalman decomposition now also apply to PCR systems. These procedures use two grammians as an input [10], [11]. Then from [3] observe that four different conventional Kalman decompositions may be computed from either:

1) $\left.\left.\left.W_{t_{0}, t}, M_{t, t_{N}}, 2\right) W_{t_{0}, t}, M_{t_{0}, t}, 3\right) W_{t, t_{N}}, M_{t, t_{N}}, 4\right) W_{t, t_{N}}, M_{t_{0}, t}$

Procedure 1) decomposes the system at time $t \in \mathrm{T}$ into states that are a) reachable at time $t$ and unobservable at time $t$ b) reachable at time $t$ and observable at time $t \mathrm{c}$ ) unreachable at time $t$ and unobservable at time $t \quad \mathrm{~d}$ ) unreachable at time $t$ and observable at time $t$. Procedure 2) does the same as 1) with "observable at" replaced by "reconstructable from". Procedure 3) does the same as 1) with "reachable at" replaced by "controllable from". Procedure 4) does the same as 1) with "reachable at" replaced by "controllable from" and "observable at" by "reconstructable from". In each case the system structure is of the following form,

$$
\begin{aligned}
& x^{\prime}(t)= {\left[\begin{array}{llll}
x_{a}^{\prime T}(t) & x_{b}^{\prime T}(t) & x_{c}^{\prime T}(t) & x_{d}^{\prime T}(t)
\end{array}\right]^{T}, } \\
& x_{a}^{\prime}(t) \in R^{n_{a}}, x_{b}^{\prime}(t) \in R^{n_{b}}, x_{c}^{\prime}(t) \in R^{n_{c}}, x_{d}^{\prime}(t) \in R^{n_{d}}, \\
& A^{\prime}(t)=\left[\begin{array}{cccc}
A_{a a}^{\prime}(t) & A_{a b}^{\prime}(t) & A_{a c}^{\prime}(t) & A_{a d}^{\prime}(t) \\
0 & A_{b b}^{\prime}(t) & 0 & A_{b d}^{\prime}(t) \\
0 & 0 & A_{c c}^{\prime}(t) & A_{c d}^{\prime}(t) \\
0 & 0 & 0 & A_{d d}^{\prime}(t)
\end{array}\right], \\
& B^{\prime}(t)=\left[\begin{array}{llll}
B_{a}^{\prime T}(t) & B_{b}^{\prime T}(t) & 0 & 0
\end{array}\right]^{T}, \\
& C^{\prime}(t)=\left[\begin{array}{llll}
0 & C_{b}^{\prime}(t) & 0 & C_{d}^{\prime}(t)
\end{array}\right], t \in\left(t_{i}, t_{i+1}\right), \\
& i=0,1, . ., N-1 .
\end{aligned}
$$

\section{Theorem 1}

1) Using our definition of PCR systems (3) the system decompositions (11) may be interpreted as decompositions into four PCR sub-systems defined over $\mathrm{T}$ having timevarying dimensions in general. Over each time interval $\left(t_{i}, t_{i+1}\right), i=0,1, . ., N-1$, the number of states $n_{i}, n_{a}, n_{b}$, $n_{c}, n_{d}$ of the PCR system and sub-systems are constant.

2) The input-output map of a PCR system is solemnly determined by PCR sub-system b) generated by Kalman decomposition 1). This sub-system is a minimal realization of the PCR system if $x\left(t_{0}^{+}\right)=0$. If $x\left(t_{0}^{+}\right) \neq 0$ the reachability grammian $W_{t_{0}^{+}, t}$ should be replaced by the so-called weak reachability grammian $W_{t_{0}^{+}, t}^{\prime}$ that is also described by equations (7), (8) except for the initial condition $W_{t_{0}^{+}, t_{0}^{+}}^{\prime}=x\left(t_{0}^{+}\right) x\left(t_{0}^{+}\right)^{T}$.

\section{Proof}

1) Conventional constant rank systems have constant dimensions, grammians with boundary conditions equal to zero and the property that the four Kalman decompositions produce sub-systems having equal and constant dimensions [6]. For PCR systems the boundary conditions of the grammians over each time interval $\left(t_{i}, t_{i+1}\right), i=1,2, \ldots, N-2$, are non-zero in general. They affect the dimensions of the sub-systems obtained from the four Kalman decompositions, which therefore can no longer be guaranteed equal. But they do not change the fact that these sub-systems have constant dimensions.

2) This follows from definitions 2 and 3 and application of the results in [6] over each separate interval $\left(t_{i}, t_{i+1}\right)$, $i=0,1, . ., N-1$. For $x\left(t_{0}^{+}\right) \neq 0$ the result follows from [12]

Now, to introduce the differential Kalman decomposition and its importance, reconsider example 1. Observe that, according to definitions 4 and 5, the system is reachable at any time $t$, controllable from any time $t$, observable at any time $t$ and reconstructable from any time $t$. After applying a similarity transformation therefore, the four Kalman decompositions will generally not reproduce the system structure of example 1 for $t \in(0.25,0.75)$. The following lemma that follows immediately from definitions 4,5 and lemma 2 applies to the time-intervals $(0.25,0.5)$ and $(0.5,0.75)$ of example 1 respectively.

\section{Lemma 3}

A PCR system being d-unreachable over $\left(t_{i}, t_{i+1}\right)$ is equivalent with the PCR system being d-uncontrollable over $\left(t_{i}, t_{i+1}\right)$ for some $0 \leq i \leq N-1$. A dual result applies to d-unobservability and d-unreconstructability.

So what has been termed temporal unreachability/ uncontrollability in section II is now formalized as being $\mathrm{d}$ uncontrollable/d-unreachable over $\left(t_{i}, t_{i+1}\right)$ for some $0 \leq i \leq N-1$. A dual result applies with respect to $\mathrm{d}$ unobservability/d-unreconstructability. So now the question is: can we device a Kalman decomposition based on dreachability/d-controllability and d-observability/dreconstructability? This type of Kalman decomposition has actually already been presented in [6]. In this paper we call it the differential Kalman decomposition. In [6] this decomposition is defined only for conventional constant rank systems. According to lemma 2 these systems are 
either d-controllable everywhere on their time domain or nowhere [6]. Therefore they cannot be temporarily uncontrollable/unreachable. This reveals that the description and detection of temporal uncontrollability requires $P C R$ systems!

\section{Definition 7}

The $d$-reachability/d-controllability grammian $W_{t}$ of a PCR system at every time $t \in \mathrm{T}$ is given by,

$W_{t}=C_{j}(t) C_{j}^{T}(t), C_{j}(t)=\left[\begin{array}{llll}P_{0}(t) & P_{1}(t) & . . & P_{j}(t)\end{array}\right]$,

$P_{0}(t)=B(t), P_{i+1}(t)=-A(t) P_{i}(t)+\dot{P}_{i}(t)$,

$i=0,1, . ., j-1$,

with $j$ the smallest value for which $\operatorname{rank}\left(C_{j+1}(t)\right)=$ $\operatorname{rank}\left(C_{j}(t)\right)$.Dually the $d$-observability/d-reconstructability grammian $M_{t}$ of the PCR system at time $t$ is given by,

$M_{t}=O_{k}^{T}(t) O_{k}(t), O_{k}=\left[\begin{array}{llll}S_{0}^{T}(t) & S_{1}^{T}(t) & . . & S_{k}^{T}(t)\end{array}\right]^{T}$,

$O_{0}(t)=C(t), O_{i+1}(t)=O_{i}(t) A(t)+\dot{O}_{i}(t)$,

$i=0,1, . ., k-1$,

with $k$ the smallest value such that $\operatorname{rank}\left(O_{k+1}(t)\right)=$ $\operatorname{rank}\left(O_{k}(t)\right)$.

\section{Definition 8}

The differential Kalman decomposition at very time $t$ uses as an input the $d$-reachability/d-controllability grammian $W_{t}$ and the $d$-observability/d-reconstructability grammian $M_{t}$ of the PCR system.

\section{Theorem 2}

1) The differential Kalman decomposition at every time $t \in \mathrm{T}$ decomposes a PCR system according to (11) into states that are a) d-reachable/d-controllable and dunobservable/d-unreconstructable at time $t$ b) d-reachable /d-controllable and d-observable/d-reconstructable at time $t$ c) d-unreachable/d-uncontrollable and d-unobservable/ d-unreconstructable at time $t$ d) d-unreachable/duncontroll. and d-observable/d-reconstructable at time $t$.

2) Over each time interval $\left(t_{i}, t_{i+1}\right), i=0,1, . ., N-1$, the number of states $n_{a}, n_{b}, n_{c}, n_{d}$ generated by the differential Kalman decomposition is constant. So a) b) c) and d) may be regarded as PCR sub-systems of the PCR system. Like the original PCR system they have constant dimensions over $\left(t_{i}, t_{i+1}\right), i=0,1, . ., N-1$.

\section{Proof}

Identical to the proof of lemma 2
From theorem 2 the differential Kalman decomposition does reproduce the system structure of example 1 after a similarity transformation has been applied. So the differential Kalman decomposition is able to detect dunreachability/d-uncontrollability over a time-interval as well as d-unobservability/d-unreconstructability.

\section{Theorem 3}

The d-reachability/d-controllability grammian $W_{t}$ of a PCR system at every time $t \in\left(t_{i}, t_{i+1}\right)$ may be interchanged with the controllability/reachability grammian $W_{t_{i}^{+}, t}$ with "initial condition" $W_{t_{i}^{+}, t_{i}^{+}}=0$ or "terminal condition" $W_{t_{i+1}^{-}, t_{i+1}^{-}}=0$ to obtain Kalman decompositions with identical dimensions $n_{a}, n_{b}, n_{c}, n_{d}$. A dual result holds for the dobservability/d-reconstructability grammian $M_{t}$.

\section{Proof}

From the proof of lemma 2 conventional constant rank systems have the property that they are either dreachable/d-controllable at any time or at no time [6]. But this implies that conventional constant rank systems are either "reachable at" as well as "controllable from" any time or no time. So for conventional constant rank systems interchanging the grammians does not affect the dimensions $n_{a}, n_{b}, n_{c}, n_{d}$. Over each time-interval $\left(t_{i}, t_{i+1}\right)$, $i=0,1, . ., N-1$, a PCR system is comparable to a conventional constant rank system except for the possibly non-zero initial and terminal values of the grammians (see the proof of theorem 1). Therefore these initial and terminal values should be taken equal to zero

$\square$

\section{Corollary 1}

For a PCR system five different Kalman decompositions may be computed using the differential equations (7), (9). These five Kalman decompositions only differ with respect to their boundary conditions at $t_{i}^{+}$and $t_{i+1}^{-}$. The four conventional Kalman decompositions [3] have the following generally non-zero boundary conditions respectively 1) $W_{t_{i}^{+}, t_{0}^{+}}, M_{t_{i_{1}^{-}+1}, t_{N}^{-}}$2) $\left.W_{t_{i}^{+}, t_{0}^{+}}, M_{t_{i}^{+}, t_{0}^{+}}, 3\right) W_{t_{i+1}^{-}, t_{N}^{+}}$, $\left.M_{t_{t_{i+1}^{-}, t_{N}^{-}}}, 4\right) W_{t_{i_{1}+1}^{-}, t_{\bar{N}}^{-}}, M_{t_{i}^{+}, t_{0}^{+}}$. These boundary conditions satisfy the transition rules (8), (10). They transfer global reachability, controllability, observability and reconstructability properties from one open time-interval $\left(t_{i}, t_{i+1}\right)$ to the next. Over $\left(t_{i}, t_{i+1}\right)$ the dimensions $n_{a}, n_{b}, n_{c}, n_{d}$ are constant but may be different for each of the four conventional Kalman decompositions.

The differential Kalman computes local d-reachability/dcontrollability and d-observability/d-reconstructability properties. Knowing $t_{i}, i=1,2 . ., N-1$, it may be computed in the same manner from zero boundary conditions. Then 
the four decompositions become effectively one since they do produce identical values $n_{a}, n_{b}, n_{c}, n_{d}$ over every interval $\left(t_{i}, t_{i+1}\right), i=0,1, . ., N-1$.

Suppose we start from a system description over $\left[t_{0}, t_{N}\right]$ such as example 1 not knowing $t_{i}, i=1,2, . ., N-1$ while these are required to arrive at the PCR system description (3). Then the differential Kalman decomposition computed at every time $t \in\left(t_{0}, t_{N}\right)$ from the d-reachability/dcontrollability and d-observability/d-reconstructability grammians is the only Kalman decomposition capable of detecting $t_{i}, i=1,2, . ., N-1$ as those isolated times where the structure changes, i.e. one or several of $n_{a}(t), n_{b}(t), n_{c}(t), n_{d}(t)$. The grammians require the $\mathrm{d}-$ controllability and d-reconstructability matrices $C_{j}(t)$, $O_{k}(t)$ in equations (12), (13). They in turn require knowledge of a sufficient number of derivatives of $A(t)$, $B(t)$ and $C(t)$. Now $t_{i}, i=1,2, . ., N-1$, are also precisely those isolated times where formally the differential Kalman decomposition does not exist because some of these derivatives do not exist.

\section{Example 2: Minimal realizations}

Consider example 1 converted to fit the PCR system description (3), i.e. with $t_{1}=0.25, t_{2}=0.5, t_{3}=0.75, A_{1}=$ $A_{2}=A_{3}=I$. One easily sees and computes $\operatorname{rank}\left(W_{t_{0}^{+}, t}\right)=2$, $\operatorname{rank}\left(M_{t, t_{N}^{\bar{N}}}\right)=2, t \in(0,1)$. Therefore the system is minimal despite the temporal uncontrollability / unreachability and unreconstructability/unobservability. The associated states may not be dropped because e.g. over $(0.25,0.5)$ the second d-uncontrollable/d-unreachable state is non-zero in general because at $t=0.25^{+}$it is non-zero in general. This information is transferred by $W_{0^{+}, 0.25^{+}}$that satisfies $\operatorname{rank}\left(W_{0^{+}, 0.25^{+}}\right)=2$.

Consider example 1 but with the system descriptions on the intervals $[0,0.25],(0.25,0.5]$ swapped as well as those on the intervals $(0.5,0.75],(0.75,1]$. One then easily computes $\operatorname{rank}\left(W_{t_{0}^{+}, t}\right)=1, \quad t \in(0,0.25), \quad \operatorname{rank}\left(W_{t_{0}^{+}, t}\right)=2$, $t \in(0.25,1), \quad \operatorname{rank}\left(M_{t, t_{\bar{N}}}\right)=2, \quad t \in(0,0.75), \quad \operatorname{rank}\left(M_{t, t_{\bar{N}}}\right)=1$, $t \in(0.75,1)$ and the associated minimal realization,

$$
\begin{aligned}
& A(t)=1, B(t)=1, C(t)=1, t \in(0,0.25), \\
& A(t)=\left[\begin{array}{ll}
1 & 2 \\
1 & 1
\end{array}\right], B(t)=\left[\begin{array}{l}
1 \\
0
\end{array}\right], C(t)=\left[\begin{array}{l}
1 \\
0
\end{array}\right]^{T}, t \in(0.25,0.5),
\end{aligned}
$$

$$
\begin{aligned}
& A(t)=\left[\begin{array}{ll}
1 & 1 \\
2 & 1
\end{array}\right], B(t)=\left[\begin{array}{l}
1 \\
0
\end{array}\right], C(t)=\left[\begin{array}{l}
1 \\
0
\end{array}\right]^{T}, t \in(0.5,0.75), \\
& A(t)=1, B(t)=1, C(t)=1, t \in(0.75,1) \\
& A_{1}=\left[\begin{array}{ll}
1 & 0
\end{array}\right]^{T}, A_{2}=I, A_{3}=\left[\begin{array}{ll}
1 & 0
\end{array}\right]
\end{aligned}
$$

Note that the minimal realization (14) has time-varying state-dimensions since over $(0,0.25)$ the second state is unreachable and over $(0.75,1)$ the second state is unobservable.

\section{CONCLUSIONS}

The introduction of PCR systems together with the differential Kalman decomposition enables the description and detection of local, temporal system structure of timevarying linear systems. This structure is associated with dreachability/d-controllability and d-observability/dreconstructability. It reveals the temporal loss of the associated global system properties reachability, controllability, observability and reconstructability. This is highly relevant to control engineers. Moreover this paper reveals that the differences between reachability versus controllability and observability versus reconstructability are entirely due to changes of the local system structure. Finally the time-varying PCR system dimensions enable the well rounded realization theory suggested by Kalman [4].

\section{REFERENCES}

[1] M. Athans, 1971, "The role and use of the Linear- QuadraticGaussian problem in control system design", IEEE Trans. Aut. Contr., 16, 529-552.

[2] L.G. Van Willigenburg, 1990, "First-order controllability and the time optimal control problem for rigid articulated arm robots with friction", Int. J. Contr., 51, 6, pp. 1159-1171

[3] L. Weiss, R.E. Kalman, 1965, Contributions to linear systems theory, Int. J. Eng. Sc., 3, pp. 141-171.

[4] R.E. Kalman, P.L. Falb, M. Arbib, 1969, Topics in Mathematical Systems Theory, Mc Graw Hill, New York.

[5] H. Sandberg, A. Rantzer, 2004, "Balanced truncation of linear timevarying systems", IEEE Trans. Aut. Contr., 49, 2, pp. 217-229.

[6] L.M. Silverman, H.E. Meadows, 1969, "Equivalent realizations of linear systems", SIAM J. Appl. Math., 17, 2, pp.393-408.

[7] G. Xie, D.Zheng, L. Wang, 2002, "Controllability of switched linear systems", IEEE Trans. Aut. Contr., 47, 8, pp. 1401-1405.

[8] G. Stikkel, J. Bokor, S. Szabo, 2004, Necessary and sufficient cond. of switching linear hybrid systems, Automatica, 40, 6, 1093-1098.

[9] I. Gohberg, M.A. Kaashoek, L. Lerer, 1992, "Minimality and realization of discrete time-varying systems", Operator theory: Advances and applications, 56, 261-296. Birkhauser.

[10] R.E. Kalman, 1963, "Mathematical description of linear systems", SIAM J. Contr, A, 1, 2, pp.152-192.

[11] D. Boley, 1984, "Computing the Kalman decomposition: An optimal method", IEEE Trans. Aut. Contr., AC-29, 1, pp. 51-53.

[12] L.G. Van Willigenburg, W.L. De Koning, 2002, "Minimal and nonminimal optimal fixed-order compensators for time-varying discretetime systems", Automatica, 38, pp.157-165. 